\title{
About the measurements of the hard X-ray background
}

\author{
G.S. Bisnovatyi-Kogan and A.S. Pozanenko \\ Space Research Institute (IKI), Moscow, Russia, Profsoyuznaya 84/32, Moscow 117810, \\ Russia \\ gkogan@iki.rssi.ru
}

Received __; accepted _ _ 


\begin{abstract}
We analyze uncertainties in the cosmic X-ray background measurements performed by the INTEGRAL observatory. We find that the most important effect limiting the accuracy of the measurements is related to the intrinsic background variation in detectors. Taking into account all of the uncertainties arising during the measurements we conclude that the X-ray background intensity obtained in the INTEGRAL observations is compatible with the historic X-ray background observations performed by the HEAO-1 satellite.
\end{abstract}

Subject headings: diffuse X-ray background; X-ray sources 


\section{Introduction}

Since the discovery of the cosmic X-ray background (CXB) in the early X-ray observations (Giacconi et al., 1962) the importance of the CXB measurement was recognized. The CXB can be considered as an indicator of the activity of unresolved active galactic nuclei (AGN). The spectral shape of the CXB and its normalization near CXB peak (20-40 keV) is particularly important for estimation of the population of Compton thick AGN, which are difficult to resolve at lower energies, because of their high intrinsic

absorption (Ueda et al., 2003; Gilli et al., 2007; Sazonov et al., 2008; Treister et al., 2009).

Measurement of CXB is a sophisticated procedure requiring a knowledge of different factors contaminating CXB. The omnidirectional detectors used in the pioneering X- and gamma-ray observations by KONUS experiment permitted to investigate CXB in the energy range above $30 \mathrm{keV}$ (Mazets, et al., 1974). More detailed CXB observations can be performed by aperture telescopes. However, contaminating factors such as an intrinsic detector background, still require either precise modelling, or several observations with different relative contributions of the intrinsic background and CXB. The latter technique was used in early measurements of the hard X-ray background in the range $13-180 \mathrm{keV}$ onboard HEAO-1 (Gruber et al., 1999).

Intrinsic background contribution in the HEAO-1 CXB experiment was measured with the detector aperture blocked. Subtracting the intrinsic instrument background from the total flux, measured with the open aperture permitted the estimate of the spectrum of the hard X-ray background (Fig. 1-2). Sky-looking data of 224 ks total duration, and 205 ks of observation was collected with the aperture closed when only the intrinsic detector background was recorded.

In the new CXB measurements performed by the INTEGRAL observatory (Churazov et al., 2007; Türler et al., 2010), Beppo-Sax (Frontera et al., 2007), and BAT/Swift (Ajello 


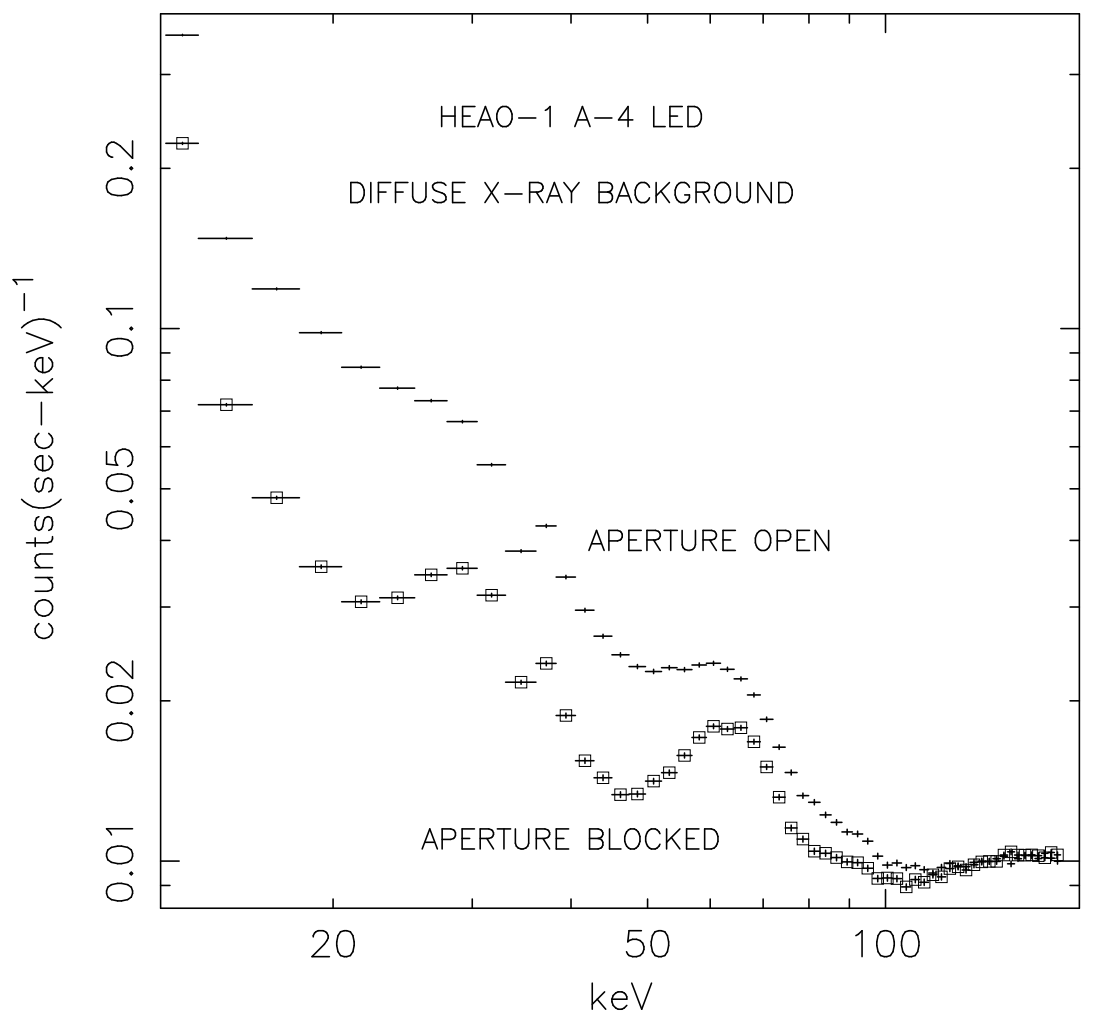

Fig. 1. - The differential counting rates obtained by the Low Energy Detectors on the UCSD/MIT Hard X-ray and Gamma-Ray Instrument on the High Energy Astronomical Observatory (HEAO-1). The difference between the rates with the detector blocked by an active shutter, and unblocked when looking at the sky well above the horizon, is due to the diffuse component of the cosmic X-rays. The rates are averaged over the similar ranges of B,L magnetic coordinates. The artifact at about $32 \mathrm{keV}$ is due to the energy-loss anomaly in $\mathrm{NaI}$ near the K-edge. The diffuse flux is well above the detector background to at least $100 \mathrm{keV}$; from Gruber et al.(1999). 


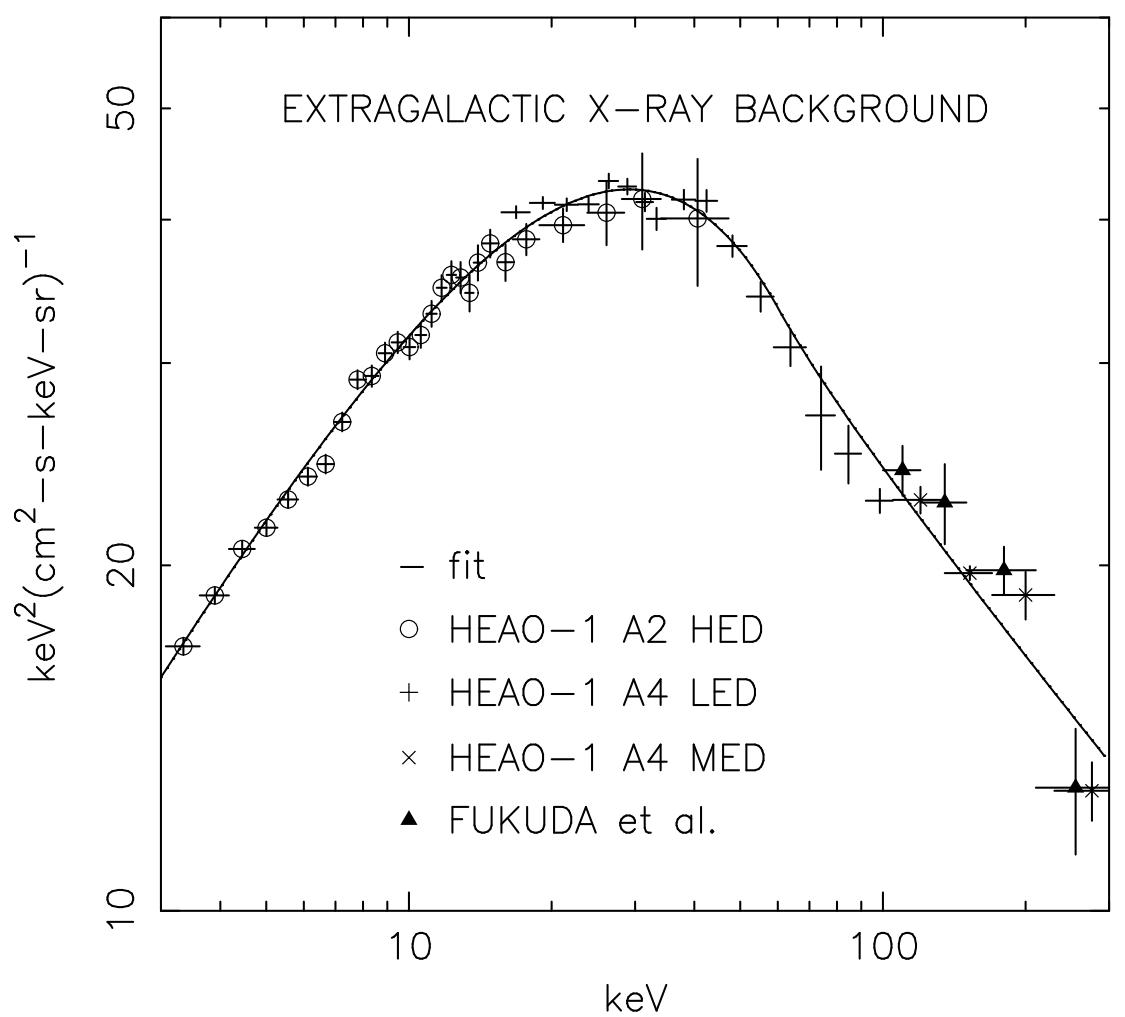

Fig. 2.- Corrected photon spectrum of the diffuse components measured by the HEAO-1 by several different detectors, in comparison with other data in this energy range, expressed as spectral intensity per logarithmic energy unit dI/d(ln E). The HEAO 1 A-4 Low Energy Detectors join smoothly to other data at both higher and lower energies, and are in agreement with balloon data, for which that of Fukada et al. (1975) has been chosen as representative. The fit is shown as a curved line. The data from HEAO-1 A2 were taken from High-Energy Detector no. 1. These A2 points may reflect minor artifacts of the spectral inversion; from Gruber et al. (1999). 
et al., 2008) the Earth was used as a natural block, and a special procedure was used to get rid of the intrinsic instrument background, based on the observations of CXB modulated by the Earth in FOV of the telescopes.

In 2006 the INTEGRAL observatory (Winkler et al., 2003) performed four runs of $\sim 30$ ksec observations with the Earth disk crossing the field of view of the JEM-X, IBIS/ISGRI and SPI telecopes (Churazov et al., 2007, hereafter CE2007). Indeed the Earth is not a perfect block, and additional estimations of a non-background X-ray emission were completed: estimation of the Earth albedo (i.e. the true CXB reflection by Earth atmosphere) (Churazov et al, 2008), and modelling of the proper emission of the Earth atmosphere resulting from a bombardment by the cosmic rays (Sazonov et al., 2007). As a reference point the spectrum obtained early by the HEAO-1 observations (Gruber at al, 1999) was used. No attempt has been made to revise the spectral shape, and only the normalization of the spectral curve was verified. The CE2007 claims that "The observed flux near the peak of the CXB spectrum is $\sim 10 \%$ higher than suggested by Gruber et al., 1999.", see Fig. 3.

A different approach was used in the CXB estimations by Türler et al. 2010 (hereafter TM2010). The authors did not fix the form of the CXB spectrum, while fitting different components (true CXB, Earth albedo, and Earth emission), using count rate of IBIS/ISGRI during the Earth occultation observations. Based on the same data used in CE2007 they concluded that the CXB normalization is fully compatible with the HEAO-1 observations (Gruber at al, 1999).

Considering different CXB normalization obtained in the same INTEGRAL observations, we discuss factors which influence on the precision of the measurement of CXB by occultation technique: the variability of the intrinsic background induced by the energetic particles along the orbit of the INTEGRAL observatory, compact X-ray 
sources reflected by the Earth atmosphere, variability induced by the non-resolved compact $\mathrm{X}$-sources in the field of view of the telescope. The last two factors have been already estimated in CE2007, and we revise the estimates. In CE2007 the intrinsic background in the detectors was assumed to be constant, while the actual background level varies along the orbit and introduces an additional uncertainty in the CXB measurement. In TM2010 the intrinsic background variation was taken into account by simple tracing of the IBIS/ISGRI background via SPI-ACS count rate. In TM2010 the uncertainties induced by the proper Earth emission due to the cosmic rays, as well as specific uncertainties due to Galactic ridge X-ray in FOV have also been considered.

\section{Reflection of X-ray sources emission by the Earth atmosphere}

The Earth atmosphere reflects X-rays from both CXB and compact sources. Reflected emission contaminates measurement of CXB when the Earth is used as a block. Indeed the CXB reflection dominates over the Galactic sources reflection. The contribution of the emission from the Crab nebula (the most intense Galactic source at $30 \mathrm{keV}$ ), reflected by the Earth, in comparison with CXB, was estimated as low as 1\% (CE2007). Using the same approach we analyze the contribution of other compact X-ray sources.

Sources behind the Earth do not give any input, and for the source in front of the Earth the effective illuminated square is equal to $\pi R_{\oplus}^{2}$. When the angle $\theta$ between the directions to the Earth and to the source is in the range $0<\theta<\pi$, the effective square for the reflected radiation which illuminates the Earth and falls onto the instrument, is equal to $\pi(1-\cos \theta) R_{\oplus}^{2} / 2(\theta=0$ corresponds to the source behind the Earth). We analyze reflection of different X-ray sources, in comparison with the Crab, taken into account the geometrical factor $(1-\cos \theta) / 2$. 
Using the sources in 20 - $40 \mathrm{keV}$ energy band included in the BATSE occultation catalog (Harmon et al., 2004) we estimate the relative contribution of the reflected emission from the sources of the catalog in comparison with the Crab nebula. The relative contribution of the reflected emission of Cyg X-1 to the Crab nebula is equal to $\sim 545 / 900$, and the relative (to the Crab) contribution of all sources of the catalog with the flux larger than $1 \mathrm{mCrab}$ is equal to $\sim 2123 / 900=2.4$. Therefore the total uncertainties due to reflection of the compact sources in $20-40 \mathrm{keV}$ energy band is $\sim 3.4$.

In the energy band 2-10 keV similar estimates can be done using BeppoSax catalog (Verrecchia et al. 2007). In this energy band the Sco X-1 gives the main contribution into the reflected flux from the Earth atmosphere. With account of the geometrical factor the contribution of the Sco X-1 flux relative to the Crab is $3765 / 1864$, and for all sources in the sky this ratio is equal to $9212 / 1864=4.9$.

Assuming the contamination by the Crab at $40 \mathrm{keV}$ as $1 \%$, one can estimate the contamination by the other sources as $2.4 \%$. Hence total uncertainty in CXB measurement induced by compact sources at $40 \mathrm{keV}$ is $\sim 3.4 \%$. The contamination at the energies $<10 \mathrm{keV}$ is significantly less. Taking the albedo of the Earth atmosphere at $10 \mathrm{keV}$ (40 keV) equals to 0.01 (0.3) (Churazov et al., 2008), one can estimate the uncertainty induced by the compact sources as $4.9 \times \frac{0.01}{0.3} \%=0.2 \%$. Using the above values one should bear in mind, that these estimates were obtained for a model in which the spectrum of each compact source is supposed to have the same spectral shape as the CXB spectrum.

\section{Variability of the intrinsic background in detector}

The time dependent spectral distribution of the X-ray flux measured by the INTEGRAL instruments, with the Earth in the field of view, was approximated by the following formula 
(CE2007),

$$
\begin{array}{r}
F(E, t) \approx C(E)-S_{\mathrm{CXB}}(E)[1-A(E)] \Omega(t)+ \\
S_{\mathrm{ATM}}(E) \Omega(t)=C(E)-S_{\mathrm{Earth}}(E) \Omega(t) .
\end{array}
$$

Here $S_{\mathrm{ATM}}(E)$ is the spectrum of the cosmic ray induced atmospheric emission (averaged over the Earth disk and normalized per solid unit angle), and $S_{\text {Earth }}(E)=$ $S_{\mathrm{CXB}}(E)[1-A(E)]-S_{\mathrm{ATM}}(E)$ is the combined flux of all components related to the presence of the Earth in the FOV, and A(E) is the energy dependent albedo of the Earth atmosphere. The term $C(E)$ includes the intrinsic background of the detector, the total combined flux of Galactic sources, and CXB. The term $C(E)$ is considered as a constant in the paper CE2007. We show here that the variability of the intrinsic background induced by the energetic particles along the orbit should introduce a most significant uncertainty in the CXB measurement.

\subsection{Background evolution in detectors}

The background included into the term $C(E)$ of the equation (1) contains at least 4 components:

1. Excitation of the matter of detectors and surrounding material by energetic protons, and subsequent radiative decay of excited isotopes (line-like emission). This decay leads to the retarded increase of the background with a characteristic time, determined by the half-decay times of the excited isotopes.

2. The intrinsic background induced by charged particles (electrons and nuclei) instantly interacting with detectors (continuum emission).

3. The true CXB from the regions on the sky never occulted by the Earth. 
4. Resolved and unresolved Galactic and extragalactic sources.

\subsection{Radiation belts}

The charged particles at the INTEGRAL are monitored by the IREM instrument (Hajdas et al., 2003). According to the IREM, the satellite crosses the outer radiation belt containing electrons, up to the distance $\sim 7 R_{\oplus}$, during $7-10$ hours. Along the orbit the flux of electron with energies $E_{e}>0.5 \mathrm{MeV}$ is decreasing during this time from $F_{e}=10^{7} \mathrm{~cm}^{-2} \mathrm{~s}^{-1}$ up to the unperturbed flux $F_{e}=500 \mathrm{~cm}^{-2} \mathrm{~s}^{-1}$. The inner radiation belt consists of protons. As the satellite crosses this belt during $\sim 1.5$ hours, the proton density is decreased $\sim 5$ times, from $F_{p}=10 \mathrm{~cm}^{-2} \mathrm{~s}^{-1}$ down to $F_{p}=2 \mathrm{~cm}^{-2} \mathrm{~s}^{-1}$ in the unperturbed state (Hajdas et al., 2003). Obviously the influence of the inner radiation belt on the intrinsic background results mostly from the isotope decay with a half-decay time of the order of few hours.

As we will see from the consideration of SPI-ACS count rate (sect. 3.3), the influence of the internal radiation belt on the background continuum is negligible. Indeed the SPI-ACS background variations do not possess a regular decay pattern after the belt passing. However the tail of the outer radiation belt may influence on the detectors far away (see sect. 3.3)

\subsection{SPI and SPI/ACS background}

The background variations for the SPI and SPI/ACS instruments have been investigated by Jean et al. (2003). It was shown that the outer electron belt is influencing the continuum background considerably, but it does not excite the isotopes. These variations influence mainly the background at energies $<100 \mathrm{keV}$. The measurements presented by Jean et al. 
(2003) show $\sim 10 \%$ variations arising from the 27 days solar rotation period. Periodic variations correlated with the $\sim 3$ days period of the satellite revolution around the Earth are also visible. Their amplitude is estimated as $\sim 2 \%$. The authors (Jean et al., 2003) conclude that "The total background variations are weak (less than a few percent) thanks to the high eccentricity orbit and high perigee of the INTEGRAL observatory."

Due to the Anti Coincidence Shield (ACS) the absolute background level in the Ge detectors of SPI is significantly depressed. However the relative background variations are similar in both SPI and SPI/ACS (see fig. 6 of Jean et al., 2003) and the SPI/ACS

background variations can be a tracer of the variations in the Ge detectors of SPI in a wide energy band.

Particular cases of background variations in SPI-ACS during CXB observations are presented in Figure 4. Non-monotonous SPI-ACS background variations can be explained by the different geometry of the INTEGRAL orbit against the Earth's magnetopause and magnetotail due to the orbit precession. To the author's knowledge currently there is no exact model of the SPI-ACS background evolution.

\subsection{JEM-X background}

The signature of the variability of the internal background can be suggested in Fig.3 of CE2007, where the level of the $C(E)$ term before the Earth occultation is larger than after it, according to the measurements of the JEM-X. Partially it might be attributed to a stability of JEM-X gain after turning on the instrument (see section 2.3 of CE2007). Alternatively the variability can be related to the intrinsic instrument background due to changing of the radiation conditions.

The background in JEM-X was discussed by Houvelin et al. (2003). It was concluded 
that "the total background level varied with a range of approximately $5 \%$ between different orbital sections. the variation is significant, but small."

\subsection{IBIS/ISGRI background}

The IBIS/ISGRI sensitivity and background is discussed in the paper of Lebrun et al. (2005). However, to the author' knowledge, there are no investigations of the intrinsic ISGRI detector background along the INTEGRAL orbit. It was suggested by the authors of TM2010 that the intrinsic ISGRI background correlates with the SPI-ACS count rate. While in general this is a quite reasonable suggestion confirmed by the investigation of correlation of the count rate in IBIS/ISGRI and SPI-ACS during solar flare (see details in TM2010), one can take into account that spallation reactions of different chemical elements in detectors of SPI-ACS (Ge, Bi) and ISGRI (Cd, Te) may lead to a difference in the background evolution in particular energy bands.

\section{Influence of the background variations on the accuracy of CXB measurement}

One can see that the amplitudes of the intrinsic background variations along the orbit are specific for each instrument and the energy band, and can vary from $2 \%$ (sect. 3.3) up to $5 \%$ (sect. 3.4). These variations should be taken into account in the long term observations, if the required accuracy is comparable with the amplitude of the background variation. In the particular case of CXB measurement the amplitude of the intrinsic background variations should be compared with amplitude of the CXB modulation related to the Earth occultation.

The CXB modulation is maximal in energy band $20-40 \mathrm{keV}$, where the energy spectrum 
of CXB has the maximum (see Fig. 2). In this band the total effect (CXB depression due to the Earth occultation plus the Earth atmosphere emission and the Earth CXB reflection (Earth albedo) can be estimated as much as $\sim 10 \%$ of the total flux of CXB in FOV registered in SPI and IBIS/ISGRI (see e.g Fig. 7 of CE2007). The uncertainty induced by the background variation during the CXB measurement can be estimated as a ratio of the amplitude of the background variation to the amplitude of the CXB modulation. The actual amplitude of the background variation during the Earth occultation observation can be estimated as low as 1\% (see Fig. 4). As we have seen above (sect. 3.3) the background in SPI-ACS is a tracer of the background in SPI detectors. Thus, the uncertainty of the CXB measurement induced by the background variation in SPI is equal to $1 / 10=10 \%$, provided the flux corresponding to the intrinsic background is comparable to the flux of CXB in the aperture of the telescope. At least for SPI and IBIS/ISGRI the intrinsic background is comparable with CXB component in the total count rate.

The uncertainty induced by the background variations is increasing beyond the peak of the CXB spectrum because the CXB modulation is decreasing (see e.g. Ajello et al., 2008).

Accounting of the intrinsic background is an essential part of the accuracy improvement of the CXB measurements. Modelling of the intrinsic background in the CXB occultation technique is used in the latest CXB observations (e.g. Ajello et al., 2008) and new analysis of IBIS/ISGRI data (TM2010) of the Earth occultation observations in 2006 (CE2007).

\section{CXB variations due to unresolved sources}

The CXB flux measured in the telescope aperture varies due to a number of unresolved compact sources, both Galactic and extragalactic. Indeed, we do not know a priori which unresolved source belongs to the Galaxy, and which one is extragalactic. Poisson variations 
of the source number in FOV at the sensitivity of observation, lead to the uncertainty in the CXB measurement. While the sensitivity of the IBIS/ISGRI in 20-50 keV band is $f_{x} \sim 10^{-11} \mathrm{ergs}^{-2} \mathrm{sm}^{-1}$ (i.e. the sensitivity in a deep extragalactic survey (Krivonos et. al, 2005) the actual sensitivity during the CXB measurement can be estimated as

$\sim 4.5 \cdot 10^{-11} \mathrm{ergs}_{\mathrm{cm}}^{-2} \mathrm{~s}^{-1}$. This sensitivity roughly corresponds the faintest source detected in FOV of IBIS/ISGRI (see the Table 2 of CE2007). Using $[\log (N(>f))=-(3 / 2) \log (f)]$ cumulative distribution for the extragalactic sources (Krivonos et. al, 2005) one can obtain normalization of the distribution at the actual sensitivity flux level $\sim 4.5 \cdot 10^{-11} \mathrm{ergs} \mathrm{cm}^{-2} \mathrm{~s}^{-1}$ as $1.4 \cdot 10^{-3} \mathrm{deg}^{-2}$, which leads to the uncertainty of CXB measurements of about $\sim 2 \%$.

Estimates of the uncertainty due to the unresolved galactic sources is less strict due to the presence the Galactic Ridge as well as numerous galactic sources in FOV of telescopes. The normalization of cumulative distribution $[\log (N(>f))=-(1) \log (f)]$ can be estimated as the number of the sources detected during the CXB observation (equal to 11) divided by the effective FOV of IBIS/ISGRI $(\sim 15 \times 15 \mathrm{deg})$, resulting to $0.05 \mathrm{deg}^{-2}$. Thus, the the number of the galactic sources in FOV is 35 times larger than the number of the extragalactic sources, and the uncertainty of CXB measurement due to the unresolved galactic sources is negligible.

\section{Normalization of the Earth atmosphere emission}

The flux of the Earth atmosphere emission induced by cosmic rays is comparable to the CXB component, while the Earth disk in FOV of the INTEGRAL telescopes. The Earth atmosphere emission was calculated in the paper of Sazonov et al. (2007). It was shown that the photon spectrum of the Earth atmosphere emission peaks at about $44 \mathrm{keV}$, and the normalization of the approximation law is equal to $31.7 \mathrm{keV}^{2} \mathrm{~cm}^{-2} \mathrm{~s}^{-1} \mathrm{keV}^{-1} \mathrm{sr}^{-1}$ (c.f. the observed flux near the maximum of the spectrum of $\mathrm{CXB}$ at $29 \mathrm{keV}$ is 
equal to $\left.47 \mathrm{keV}^{2} \mathrm{~cm}^{-2} \mathrm{~s}^{-1} \mathrm{keV}^{-1} \mathrm{sr}^{-1}\right)$. Indeed the uncertainty of the Earth atmosphere emission should be included in the total uncertainty of the CXB measurement by the Earth occultation technique. In the procedure of CXB calculation (see equation (1), the normalization factors of both the Earth emission component, and CXB were free parameters, and the normalization of the Earth atmosphere emission was estimated as $32.9 \pm 1.3 \mathrm{keV}^{2} \mathrm{~cm}^{-2} \mathrm{~s}^{-1} \mathrm{keV}^{-1} \mathrm{sr}^{-1}$. It is well within 1 sigma confidence level of the predicted value (31.7) and leads to the $\sim 4 \%$ uncertainty of the Earth emission component determination. Therefore uncertainty of about $4 \%$ is introduced by the Earth atmosphere emission in the CXB measurements by the Earth occultation technique.

\section{Conclusion}

Summing up the uncertainties of the CXB measurement by the Earth occultation technique around CXB peak (20 - $40 \mathrm{keV})$ in INTEGRAL observations (CE2007), one can consider at least the following sources of uncertainties. The compact source reflection came as $\sim 3 \%$. The intrinsic background variability along the orbit can contribute as much as $10 \%$. Variations due to the unresolved sources at the limiting sensitivity is $\sim 2 \%$. Modelling of the atmosphere reflection $\sim 4 \%$. We should add these values to the values already discussed and estimated in the paper CE2007. Those include statistical error in the normalization of the CXB component (joint fit to JEM-X, IBIS/ISGRI, and SPI data) as $\sim 1 \%$; neglecting contribution of the compact source $4 \mathrm{U} 1626-67$ in FOV as $\leq 2 \%$; uncertainty of the Crab photon index as $\sim 1 \%$ (the accuracy of absolute calibrations of the instruments onboard the INTEGRAL observatory is also estimated as low as $\sim 1 \%$ in Jourdain et al., (2008). Combining all these uncertainties we obtain the total uncertainty in the 20-40 keV energy band as $\sim 11 \%$. One can see that the main uncertainty of the CXB measurement of the INTEGRAL observatory is induced by the intrinsic background 
variations.

A comprehensive consideration of the CXB accuracy induced by the absolute calibration, based on Crab observation in different experiments, can be found in CE2007.

Considering the precision of the recent measurements at the CXB peak $\sim 30 \mathrm{keV}$, (CE2007, Frontera et al., 2007, Ajello et al., 2008) one can see that practically all measurements are consistent within their uncertainties (see also Moretti et al., (2009)) and are comparable with the HEAO-1 observations (Gruber et al., 1999).

Note that estimates of CXB around its peak in recent BeppoSAX observation (Frontera et al. 2007; see also Frontera et al. 2008) and re-analysis of CXB obtained with the IBIS/ISGRI of the INTEGRAL (TM2010) show a good agreement with the historic HEAO-1 measurements. Meanwhile our consideration shows that the CXB normalization obtained at least for the SPI and IBIS/ISGRI detectors in CE2007 can be also compatible with the HEAO-1 measurements if we account the intrinsic background variation during the CXB measurement. We stress that modelling of the intrinsic background is necessary for increasing accuracy of any long term observations performed by the INTEGRAL observatory, and in particular in the CXB measurements.

We have not discussed several other sources of uncertainties arising in the occultation technique. These are the Earth atmosphere self-activity, such as Auroral emission, Terrestrial Gamma-ray Flashes; intrinsic variability of the X-ray sources in FOV and transient phenomena, such as Gamma-Ray Bursts, SGR, and X-ray bursters etc. While influence of some of them on the CXB measurements may be non negligible the investigation of these source is beyond the scope of this paper.

Despite decreasing statistical uncertainties in the near Earth CXB observations systematic uncertainties continue to dominate. One possible way toward improving the 
accuracy of the measurement around the CXB peak is to use the lunar orbital spacecraft and Moon as a natural block and CXB modulator. Indeed the Moon is a more perfect block with absence of any self activity (such as auroral Earth emission), and having the lowest albedo in CXB peak spectrum (Churazov et al., 2008). Moreover the absence of the magnetosphere make the lunar orbital experiments (e.g. past Lunar Prospector Gamma Ray Spectrometer, Feldman et al., 1999) suitable for the precise CXB measurement. Meanwhile, the observation of the dark side of the Moon by Chandra observatory is already used in the CXB measurement in 2-7 keV energy range (Markevitch et al., 2002).

\section{REFERENCES}

Ajello M., Greiner J., Sato G. , et al. 2008, ApJ 689, 666

Churazov E., Sunyaev R., Revnivtsev M., et al. 2007, A\&A, 467, 529

Churazov E., Sazonov S., Sunyaev R., Revnivtsev M. 2008, MNRAS 385 , 719

Feldman W.C., Barraclough B.L., Fuller K.R., et al. 1999, Nuclear Instruments and Methods in Physics Research A, 422, 562

Frontera F., Orlandini M., Landi R., et al. 2007, ApJ, 666, 86

Frontera F., Orlandini M., Landi R., et al. 2008, Chinese Journal of Astronomy \& Astrophysics Supplement, 8, 297

Fukada, Y., Hayakawa, S., Kasahara, I., et al. 1975, Nature, 254, 398

Giacconi R., Gursky H., Paolini F. R., Rossi B. B. 1962, Physical Review Letters 9 , 439

Gilli R., Comsatri A., Hasinger G., 2007, A\&A, 463, 79

Gruber D. E., Matteson J. L., Peterson L. E., Jung, G.V. 1999, ApJ, 520, 124 
Hajdas W., Bühler P., Eggel C., F et al. 2003, A\&A 411, L43

Harmon B.A., Wilson C.A., and Fishman G.J. 2004, ApJ Suppl. 154, 585

Huovelin J., Maisala S., Schultz J., et al. 2003, A\&A 411, L253

Jean P., Vedrenne G., Roques J.P., et al. 2003, A\&A 411, L107

Krivonos R., Vikhlini A., Churazov E., et al, 2005, ApJ, 625, 89

Lebrun F., Roquesc J.-P., Sauvageon A., et al., 2005, Nuclear Instruments and Methods in Physics Research A 541, 323

Markevitch M., Bautz M. W., Biller B., et al, 2003, ApJ, 583, 70

Mazets E.P., Golenetskii S.V., Il'inskii V.N., et al. 1974, JETP Letters, 20 , 32.

Moretti A., Pagani C., Cusumano G., et al. 2009, A\&A 493, 501

Sazonov S., Churazov E., Sunyaev R., Revnivtsev M. 2007, MNRAS 377, 1726

Sazonov S., Krivonos R., Revnivtsev M., et al. 2008, A\&A, 482, 517

Treister E., Urry C.M., Virani S., 2009, ApJ, 696, 110

Türler M., Chernyakova M., Courvoisier T.J.-L., et al. 2010, arXiv:1001.2110 [astro-ph.CO]

Ueda Y., Akiyama M., Ohta K., et al. 2003, ApJ, 598, 886

Verrecchia F., in 't Zand J. J.M., Giommi1 P., et al. 2007, A\&A 472, 705

Winkler C., Courvoisier T. J.-L., Di Cocco G., et al. 2003, A\&A 411, L1 


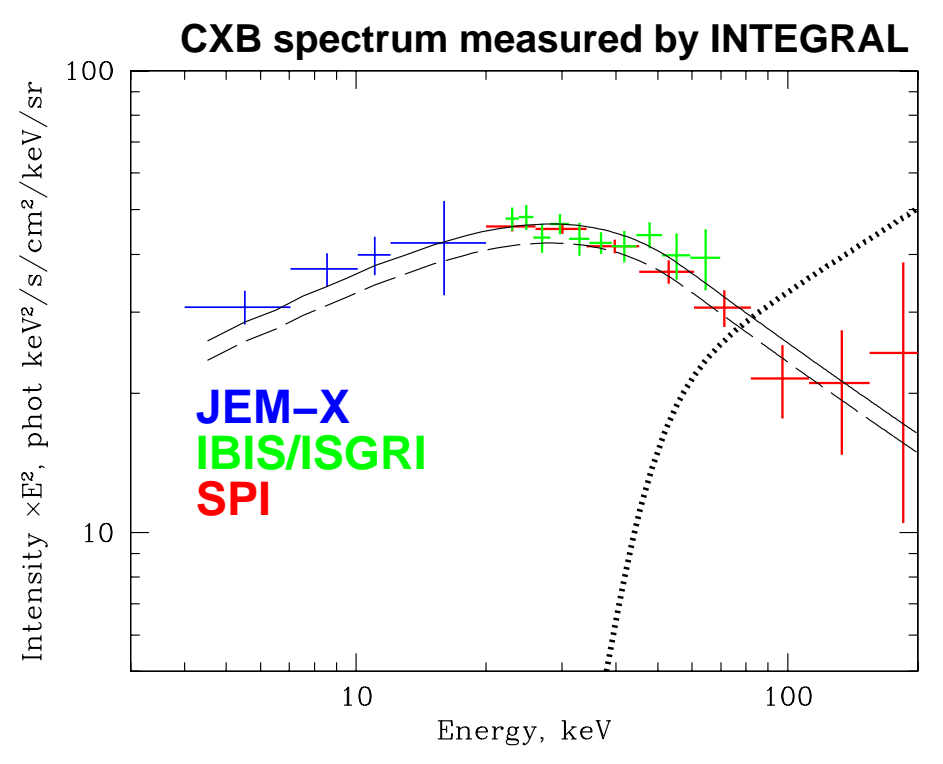

Fig. 3.- Spectrum of the CXB measured by INTEGRAL instruments. The error bars plotted account for the uncertainties in the normalization of the atmospheric emission component. The dashed line shows the analytic approximation of the CXB spectrum by Gruber et al. (1999). The solid line shows the same spectrum with the best-fit normalization obtained in the work of CE2007. The thick dotted line shows the best-fit spectrum of the Earth atmospheric emission, from CE2007. 

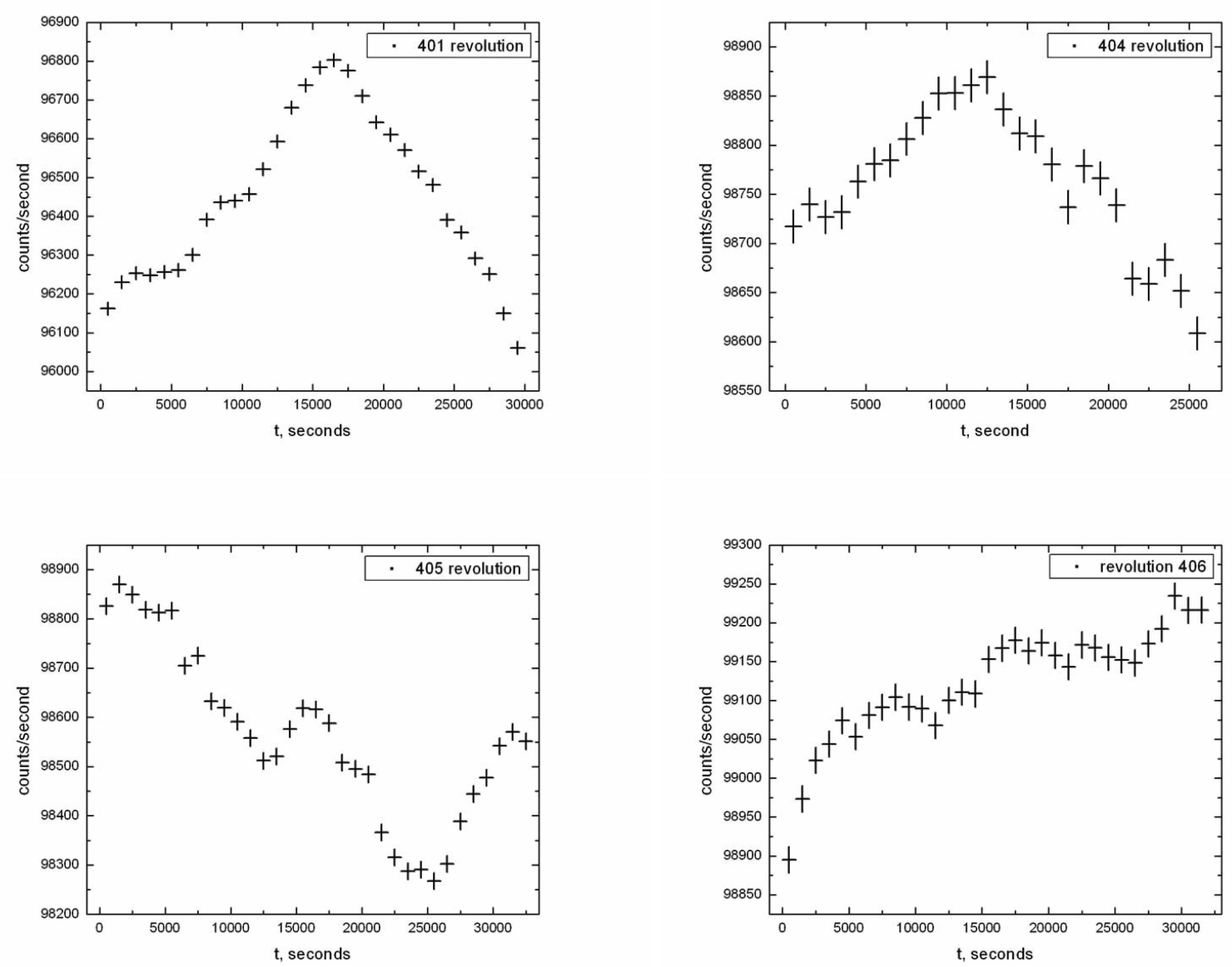

Fig. 4.- Long term SPI-ACS background evolution during CXB measurements in revolutions 401, 405 (left column), and 404, 406 (right column). Initial data of 50 ms resolution is rebinned in $1000 \mathrm{~s}$. Null in the X-axis corresponds to the switch on time of the SPI-ACS after the Earth radiation belt crossing. 